\title{
Flood Management of Lake Toke: MPC Operation under Uncertainty
}

\author{
Itsaso Menchacatorre ${ }^{1}$, Roshan Sharma ${ }^{1}$, Beathe Furenes ${ }^{2}$, Bernt Lie $^{1}$ \\ ${ }^{1}$ University of South-Eastern Norway, Porsgrunn, Norway, Bernt.Lie@usn.no \\ ${ }^{2}$ Skagerak Kraft AS, Porsgrunn, Norway
}

\begin{abstract}
A deterministic reference tracking model predictive control (MPC) is in use at Skagerak Kraft for flood management of Lake Toke in Norway. An operational inflow estimate is used to predict the optimal gate opening at Dalsfos power station, with required constraints set by the Norwegian Water Resource and Energy Directorate (NVE). The operational inflow estimate is based on the meteorological forecast, and is uncertain; this may lead to broken concession requirements and unnecessary release of water through the floodgates. Currently not utilized, the meteorological uncertainty is quantified by an ensemble of possible weather forecasts. In this paper, quantified inflow uncertainty is studied and how this affects the operation of the current, deterministic MPC solution. Next, we develop an alternative, stochastic MPC solution based on multi objective optimization which directly takes the inflow uncertainty into consideration. A comparison of the results from both approaches concludes that the stochastic MPC solution seems to give better control by reducing the amount of water released through the flood gates. Furthermore, with less frequent update of the control signal, the benefit of the stochastic MPC is expected to increase.
\end{abstract}

Keywords: model predictive control, hydrology, uncertainty, multi objective optimization

\section{Introduction}

Lake Toke in Norway, is the water magazine for five runof-river hydro power plants operated by Skagerak Kraft ${ }^{1}$. The Dalsfos hydro power plant is located at the outlet from Lake Toke, and the remaining plants are located along the Kragerø waterway, as shown in Figure 1. The Dalsfos dam has intakes to three turbines, and has two flood gates. A number of concession requirements specified by he Norwegian Water Resource and Energy Directorate (NVE) must be fulfilled to ensure safe and environmentally friendly operation. The requirements refer to constraints on change of flow out of the dam, a minimum downstream flow rate, specific seasonal lower and upper limits on the water level during the year, etc. The catchment area for Lake Toke is ca. $1150 \mathrm{~km}^{2}$, and the influent flow rate to the lake from precipitation varies considerably during the year. Skagerak Kraft subscribes to a

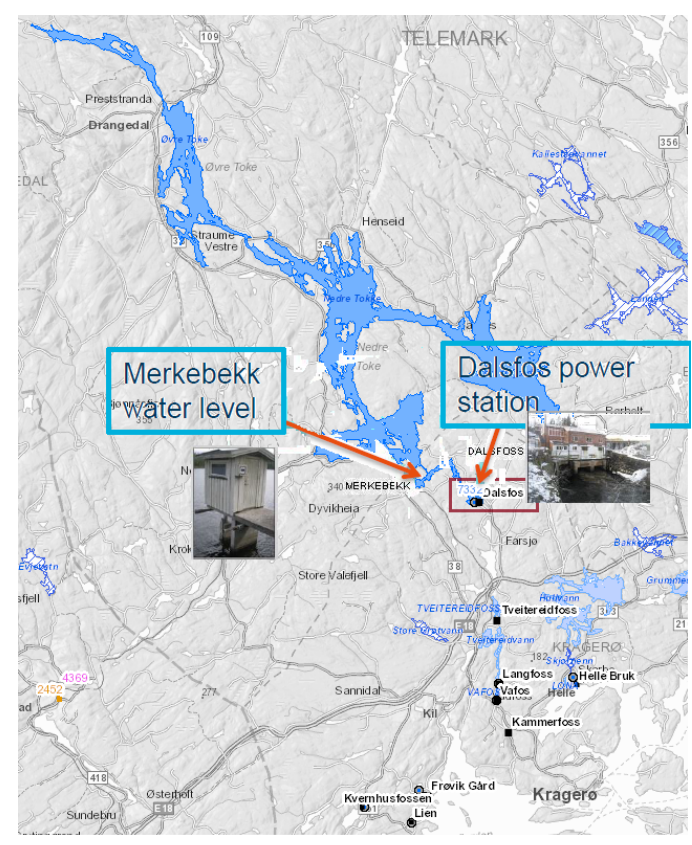

Figure 1. Overview of Lake Toke and Kragerø Waterways from (NVE, 2018).

weather forecast service provided by Storm. no, which provides updates twice a day of a most likely scenario as well as 51 possible meteorological scenarios indicating uncertainty. Based on these scenarios, Skagerak Kraft use a hydrological model to predict transport of water into Lake Toke. With uncertainty in the precipitation and the temperature, it is of interest to utilize the quantified uncertainty for improved accuracy. The use of ensemble predictions for flood control in real time is widely studied (Raso et al., 2014), (Breckpot et al., 2013a), (Breckpot et al., 2013b), including an EU project (Butts et al., 2007).

In 2014, Skagerak Kraft commissioned an MPC prototype control algorithm from $\mathrm{USN}^{2}$ to suggest flood gate openings, (Lie, 2014). Initially, the suggested opening is set manually. Based on the experience, an improved solution was developed and installed in late 2018. The implemented deterministic MPC solution is based solely on the operational forecast, and the control signal/proposed gate opening is recomputed every hour. It is now of interest to assess the deterministic MPC algorithm based on the

${ }^{2} \mathrm{USN}=$ University of South-Eastern Norway

\footnotetext{
${ }^{1}$ www.skagerakenergi.no/forside/
} 


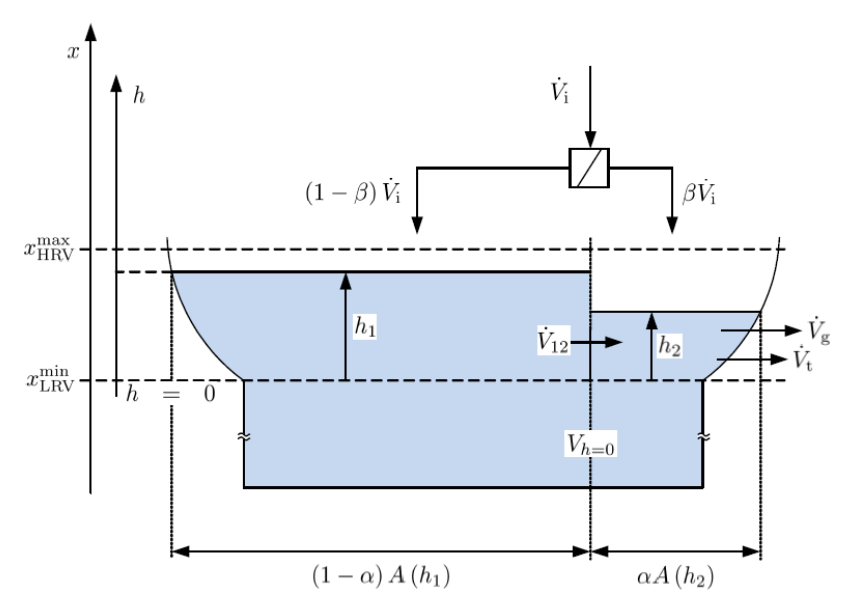

Figure 2. Assumed geometry of Lake Toke (Lie, 2014).

quantified uncertainty, and consider whether there is an advantage in using a stochastic MPC algorithm instead; (Schwanenberg et al., 2015) indicates advantages with a stochastic approach, see also (Nasir et al., 2018).

In the sequel, concession requirements and operation in flood situations are described. The paper is organized as follows. Section 2 provides a summary of the current MPC solution with model summary, relevant concession requirements, and deterministic MPC formulation. Section 3 provides an assessment of how the deterministic MPC solution handles quantified uncertainty. Section 4 develops a stochastic MPC solution, with operational results. Section 5 gives a comparison of the deterministic and the stochastic solution. Finally, some conclusions are drawn in Section 6.

\section{Current MPC solutions}

\subsection{Model Summary}

Figure 2 depicts a simplified layout of the lake, which is used as basis for a mass balance model.

Volumetric inflow $\dot{V}_{\mathrm{i}}$ to Lake Toke is a system disturbance, based on meteorological forecasts and Skagerak Kraft's hydrology model. Volumetric flow $\dot{V}_{\text {o }}$ out of Lake Toke is the sum of flow rate through turbines $\left(\dot{V}_{\mathrm{t}}\right)$ and flood gates $\left(\dot{V}_{\mathrm{g}}\right)$. Turbine flow is used to produce electric power $\dot{W}_{\mathrm{e}}$ which is scheduled daily by economists; $\dot{W}_{\mathrm{e}}$ is considered a given disturbance, and $\dot{V}_{\mathrm{t}}$ is back-computed from $\dot{W}_{\mathrm{e}}$ and other quantities. Flood gate flow rate $\dot{V}_{\mathrm{g}}$ should ideally be zero to conserve water for energy production. Because production is constrained to $\dot{V}_{\mathrm{t}} \leq 36 \mathrm{~m}^{3} / \mathrm{s}$, the flood gates may be activated in flood situations to satisfy concession requirements. The gate opening denoted $h_{\mathrm{g}}$ is the control input for the MPC. Figure 3 illustrates the operation of the floodgate, resulting in a model based on Bernoulli's law.

With disturbances $\dot{V}_{\mathrm{i}}$ and $\dot{W}_{\mathrm{e}}$, and control signal $h_{\mathrm{g}}$, a model of relevant water levels is described in (Lie, 2014). A summary of the model follows. Inflow $\dot{V}_{\mathrm{i}}$ and power production $\dot{W}_{\mathrm{e}}$ are provided by Skagerak Kraft as disturbances. States are considered to be the level offsets $h_{1}$ of

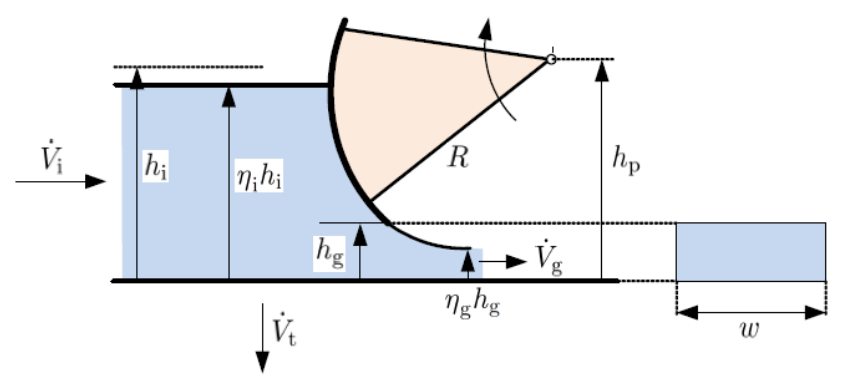

Figure 3. Assumed geometry of flood gate (Lie, 2014).

Lake Toke and $h_{2}$ in front of the dam,

$$
\begin{aligned}
\frac{\mathrm{d} h_{1}}{\mathrm{~d} t} & =\frac{1}{(1-\alpha) A\left(h_{1}\right)}\left((1-\beta) \dot{V}_{\mathrm{i}}-\dot{V}_{12}\right) \\
\frac{\mathrm{d} h_{2}}{\mathrm{~d} t} & =\frac{1}{\alpha A\left(h_{2}\right)}\left(\beta \dot{V}_{\mathrm{i}}+\dot{V}_{12}-\dot{V}_{\mathrm{t}}-\dot{V}_{\mathrm{g}}\right),
\end{aligned}
$$

with real water levels at Merkebekk Dam $x_{M}$ and Dalsfos Dam $x_{\mathrm{D}}$ expressed as

$$
\begin{aligned}
& x_{\mathrm{M}}=h_{1}+x_{\mathrm{LRV}}^{\min } \\
& x_{\mathrm{D}}=h_{2}+x_{\mathrm{LRV}}^{\min } .
\end{aligned}
$$

Area $A(h)$ is the experimentally found filling curve of the reservoir:

$$
A(h)=\max \left(28 \times 10^{6} \cdot 1.1 \cdot h^{\frac{1}{10}}, 10^{3}\right) .
$$

Inter compartment flow $\dot{V}_{12}$ is given by:

$$
\dot{V}_{12}=K_{12} \cdot\left(h_{1}-h_{2}\right) \sqrt{\left|h_{1}-h_{2}\right|} .
$$

Volumetric flow $\dot{V}_{\mathrm{t}}$ is computed from known power production $\dot{W}_{\mathrm{e}}$, and a simple turbine model involving modeled intake dam level $x_{\mathrm{D}}$ and downstream level $x_{\mathrm{q}}$ as

$$
\dot{V}_{\mathrm{t}}=a \frac{\dot{W}_{\mathrm{e}}}{x_{\mathrm{D}}-x_{\mathrm{q}}+b}
$$

where $x_{\mathrm{q}}$ is found by choosing the correct root of the cubic equation

$$
\begin{aligned}
0 & =c_{1} x_{\mathrm{q}}^{3}+\left(c_{2}-c_{1} x_{\mathrm{D}}\right) x_{\mathrm{q}}^{2} \\
& +\left(c_{3}-c_{2} x_{\mathrm{D}}+c_{4} \dot{V}_{\mathrm{g}}\right) x_{\mathrm{q}} \\
& +\dot{W}_{\mathrm{e}}-c_{3} x_{\mathrm{D}}-c_{4} \dot{V}_{\mathrm{g}} x_{\mathrm{D}}-c_{5}
\end{aligned}
$$

In reality, $x_{\mathrm{q}}$ is measured, but we need a model for $x_{\mathrm{q}}$ for predictions.

The model for flow $\dot{V}_{\mathrm{g}, j}$ through floodgate $j$ is

$$
\dot{V}_{\mathrm{g}, j}=C_{\mathrm{d}} w_{j} \min \left(h_{\mathrm{g}}, h_{2}\right) \sqrt{2 g \max \left(h_{2}, 0\right)}
$$

the two flood gates are identical except for their width $w_{j}$, and the total flood gate flow $\dot{V}_{\mathrm{g}}$ is the sum, $\dot{V}_{\mathrm{g}}=\dot{V}_{\mathrm{g}, 1}+\dot{V}_{\mathrm{g}, 2}$.

Parameters for the model are given in Table 1. 
Table 1. Parameters for Lake Toke model.

\begin{tabular}{cccl}
\hline Parameter & Value & Unit & Comment \\
\hline$\alpha$ & 0.05 & - & Fraction of surface area in compartment 2. \\
$\beta$ & 0.02 & - & Fraction of inflow to compartment 2. \\
$K_{12}$ & 800 & $\mathrm{~m}^{\frac{3}{2}} / \mathrm{s}$ & Inter compartment flow coefficient \\
$C_{\mathrm{d}}$ & 0.7 & - & Discharge coefficient, Dalsfos gates \\
$w_{1}$ & 11.6 & $\mathrm{~m}$ & Width of Dalsfos gate 1 \\
$w_{2}$ & 11.0 & $\mathrm{~m}$ & Width of Dalsfos gate 2 Comment \\
$x_{\mathrm{LRV}}^{\min }$ & 55.75 & $\mathrm{~m}$ & Minimal low regulated level value \\
$g$ & 9.81 & $\mathrm{~m} / \mathrm{s}^{2}$ & Acceleration of gravity \\
$a$ & 124.69 & $\mathrm{~Pa}^{-1}$ & coefficient, 7 \\
$b$ & 3.161 & $\mathrm{~m}$ & coefficient, 7 \\
$c_{1}$ & 0.13152 & $\mathrm{~W} / \mathrm{m}^{3}$ & polynomial coefficient, 8 \\
$c_{2}$ & -9.5241 & $\mathrm{~W} / \mathrm{m}^{2}$ & polynomial coefficient, 8 \\
$c_{3}$ & $1.7234 \cdot 10^{2}$ & $\mathrm{~W} / \mathrm{m}$ & polynomial coefficient, 8 \\
$c_{4}$ & $-7.7045 \cdot 10^{-3}$ & $\mathrm{~Pa} / \mathrm{m}$ & polynomial coefficient, 8 \\
$c_{5}$ & $-8.7359 \cdot 10^{-1}$ & $\mathrm{~W}$ & polynomial coefficient, 8 \\
\hline
\end{tabular}

\subsection{Concession Requirements}

Concession requirements are specified by NVE, and focus on (i) providing safety, (ii) securing ecological diversity, and (ii) avoiding damage to property, e.g., by maintaining certain minimum and maximum levels at Merkebekk. The key constraints for a flood situation are:

1. Abrupt changes of the water flow downstream from Dalsfos power station should be avoided for safety reasons; this is a qualitative requirement.

2. The water level $x_{M}$ of Lake Toke at Merkebekk must lie in the seasonally varying interval $x_{\mathrm{M}} \in$ $\left[x_{\mathrm{LRV}}, x_{\mathrm{HRV}}\right]$. Here $x_{\mathrm{LRV}}$ refers to the lowest regulated value and $x_{\mathrm{HRV}}$ to the highest regulated value. Level constraints are given in Figure 4.

3. In the event of a flood estimated to more than an instantaneous value of $200 \mathrm{~m}^{3} / \mathrm{s}, x_{\mathrm{M}}>x_{\mathrm{HRV}}$ is allowed until the flood has culminated; after flood culmination, $x_{M}$ is required to decrease steadily until the water level reaches $x_{\mathrm{HRV}}$ again.

4. In case of little snow in the catchment $\left(<150 \mathrm{Mm}^{3}\right)$ as the winter production comes to an end, the summer lower limit must be met at once by the reservoir. Thus, the flow should be limited to $\dot{V}_{\mathrm{o}}=\dot{V}_{\mathrm{t}}+\dot{V}_{\mathrm{g}} \leq$ $20 \mathrm{~m}^{3} / \mathrm{s}$ until the level is reached for the first time.

\subsection{Reference Tracking MPC Operation un- der Uncertainty}

Model Predictive control (MPC) is an algorithm that continuously solves an optimal control problem at each time step, with a performance measure involving a future time horizon. At each time step, only the first computed control input is used to change model states. Before each re-optimization, updated information about states is used;

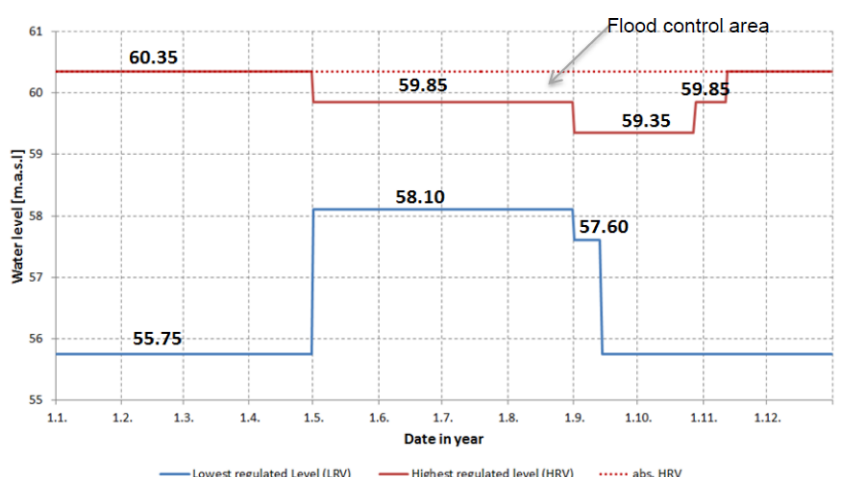

Figure 4. Level constraints in Lake Toke during the year.

this provides feedback in MPC. This MPC strategy is also known as receding horizon or sliding horizon optimal control, (Sharma, 2017). For the currently implemented MPC algorithm at Skagerak, the following quantities are relevant:

- Reference region (r): $\mathscr{R}$

- Control input $\left(u_{\mathrm{c}}\right): h_{\mathrm{g}}$

- Disturbances $\left(u_{\mathrm{d}}\right): \dot{V}_{\mathrm{i}}, \dot{W}_{\mathrm{e}}$

- Outputs $(y): x_{\mathrm{M}}, x_{\mathrm{D}}, \dot{V}_{\mathrm{t}}, \dot{V}_{\mathrm{g}}, \dot{V}_{\mathrm{o}}$

- States $(x): h_{1}, h_{2}$

The following cost function is used, (Lie, 2014),

$$
\mathscr{J}_{t}=\sum_{i=1}^{H} w_{\mathscr{R}} \mathscr{R}^{2}\left(x_{\mathrm{M}, t+i}\right)+w_{\Delta u} \Delta u_{\mathrm{c}, t+i-1}^{2}+w_{u} u_{\mathrm{c}, t+i-1}^{2}
$$

where $H$ is the length of the horizon and

$$
\Delta u_{\mathrm{c}, t+i-1} \triangleq u_{\mathrm{c}, t+i-1}-u_{\mathrm{c}, t+i-2},
$$


and subject to the dynamic model of the system:

$$
x_{i+1}=f\left(x_{i}, u_{\mathrm{c}, i}, u_{\mathrm{d}, i}\right)
$$

Expression $\mathscr{R}\left(x_{\mathrm{t}+\mathrm{i}}\right)$ is related to the level at Merkebekk in such a way that:

$$
\mathscr{R}\left(x_{\mathrm{M}, t+i}\right)=\min \left(x_{\mathrm{M}, t+i}-r_{t+i}^{\ell}, 0\right)+\max \left(x_{\mathrm{M}, t+i}-r_{t+i}^{\mathrm{u}}, 0\right) .
$$

Here $r_{t+i}^{\ell}$ and $r_{t+i}^{u}$ are lower and upper reference region boundaries, respectively. The implication of this formulation is that as long as $x_{M, t+i} \in\left[r_{t+i}^{\ell}, r_{t+i}^{\mathrm{u}}\right], \mathscr{R}\left(x_{\mathrm{M}, t+i}\right)=0$. When $x_{\mathrm{M}, t+i} \notin\left[r_{t+i}^{\ell}, r_{t+i}^{\mathrm{u}}\right]$, this is penalized in the cost function. With this in mind, the reference region boundaries are chosen based on the knowledge that emptying Lake Toke is faster than filling it, thus, being closer to $x_{\mathrm{HRV}}$ is considered less risky.

MPC provides feedback to reduce the effect of model errors and unknown disturbances, and feed-forward to take into account future reference values and known disturbances, while also handling hard constraints on variables. With many free variables in the optimization problem, solution time may be considerable. In this case, the operational forecast is 294 intervals (approximately 12d with hourly updates) and two flood gates. By constraining the two flood gate openings to one free variable and grouping the control predictions into 3 groups constant value in each group, the problem is reduced to 3 free variables. From an optimal control point of view, this is a crude approximation. However, MPC's approach of frequent recomputation of the control inputs leads to satisfactory results. In summary, every hour, a set of gate opening proposals together with predicted water levels are obtained for the specified horizon.

\section{Stochastic analysis of a determinis- tic MPC}

\subsection{Simulation principle}

The available flow rate scenarios 294 steps into the future into Lake Toke, $\dot{V}_{\mathrm{i}, 1: 294}$, consist of the operational forecast $\dot{V}_{\mathrm{i}, t}^{(\mathrm{o})}$ and 50 "particles" $\dot{V}_{\mathrm{i}, t}^{(p)}$ which can be described in a matrix,

$$
\dot{V}_{\mathrm{i}, 1: 294} \in\left(\begin{array}{cccc}
\dot{V}_{\mathrm{i}, 1}^{(\mathrm{o})} & \dot{V}_{\mathrm{i}, 1}^{(1)} & \cdots & \dot{V}_{\mathrm{i}, 1}^{(50)} \\
\vdots & \vdots & \ddots & \vdots \\
\dot{V}_{\mathrm{i}, 294}^{(\mathrm{o})} & \dot{V}_{\mathrm{i}, 294}^{(1)} & \cdots & \dot{V}_{\mathrm{i}, 294}^{(50)}
\end{array}\right) .
$$

Updated predictions are available twice daily. The morning forecast is available in the normal working hours. For simplicity, only the morning forecast is used in this study. Figure 5 depicts a forecast for the first day.

The resulting multi day forecast looks as in Figure 6.

With the deterministic MPC algorithm, the inflow prediction $u_{\mathrm{d}, i}$ used in predicting states via 11 and thus influencing the value of the cost function $\mathscr{J}_{\mathrm{t}}$ of 10 , are always

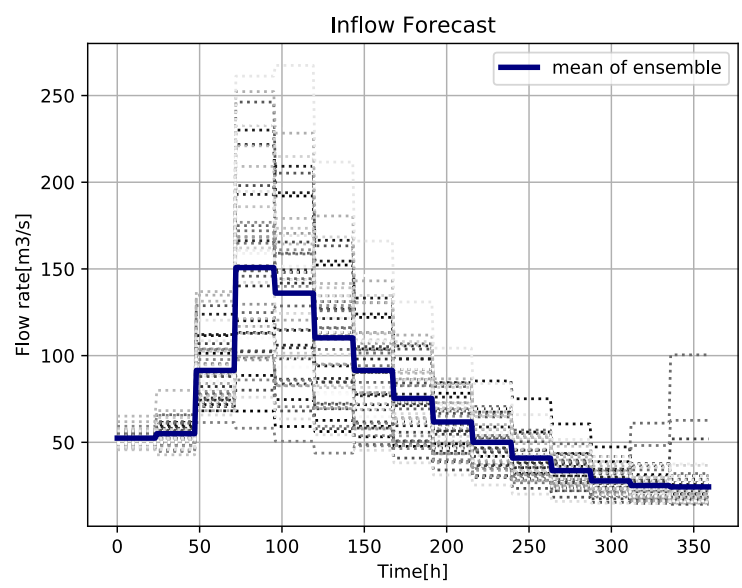

Figure 5. Inflow forecast $\dot{V}_{\mathrm{i}}$ for 294 steps into the future at Day 1 .

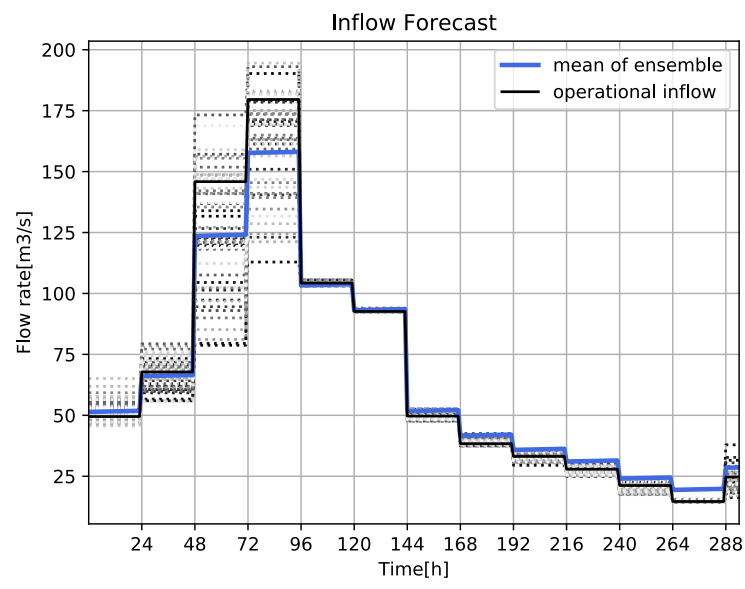

Figure 6. Inflow forecast $\dot{V}_{\mathrm{i}}$, updated every day when new forecasts are made available.

the operational values of $\dot{V}_{\mathrm{i}}$ found in the first column of the matrix in 12 . The outcome of optimizing $\mathscr{J}_{\mathrm{t}}$ is an optimal sequence of future control inputs $h_{\mathrm{g}}$; only the first of these inputs is used.

To assess how the deterministic MPC algorithm would work with the given uncertainty, in turn we consider each of the 50 additional "particles" of inflows $\dot{V}_{i}^{(p)}$ where $p \in\{1, \ldots, 50\}$ in the matrix of 12 . These 50 particles are thought to represent reality in 50 parallel "universes", while the operational inflow $\dot{V}_{i}^{(o)}$ represents the idealized model inflow. For each "universe", the inflow $\dot{V}_{\mathrm{i}}^{(p)}$ is used to update the "real" state according to 11 :

$$
x_{t+1}^{(p)}=f\left(x_{t}^{(p)}, u_{\mathrm{c}, t}^{(p)}, u_{\mathrm{d}, t}^{(p)}\right)
$$

where $u_{\mathrm{d}, t}^{(p)}=\dot{V}_{\mathrm{i}, t}^{(p)}$, while input $u_{\mathrm{c}, t}^{(p)}$ is the input computed from optimizing $\mathscr{J}_{t}$ with initial value $x_{t}^{(p)}$ and operational input predictions $\dot{V}_{\mathrm{i}, t+i}^{(\mathrm{o})}$. With $x_{t+1}^{(p)}$ computed as in $13, x_{t+1}^{(p)}$ 
is used together with operational inflow $\dot{V}_{\mathrm{i}, t+1+i}^{(\mathrm{o})}$ in optimizing $\mathscr{J}_{t+1}$, etc.

For each particle $p$ in the ensemble for $\dot{V}_{\mathrm{i}}$, we thus find trajectories of the evolution of water levels and the evolution of flood gate openings $h_{\mathrm{g}}$.

\subsection{Simulation results}

At initial time, all particles have the same initial values for the states and the control input. Thus, computed control inputs in the first time step are the same for all the particles, since the operational forecast is fed to the MPC algorithm. By contrast, the next computed states can differ from particle to particle because ensemble disturbances are introduced. For each time instance in the evolution of the 50 particles, mean and standard deviation over the particles are used to measure the uncertainty involved in the evolution of levels and flood gate opening.

During testing of the computations in Python, it is observed that optimization of the cost function $\mathscr{J}_{t}$ for a single particle at a single time instance under a flood situation can take up to $30 \mathrm{~s}$, as opposed to $2 \mathrm{~s}$ if the optimal solution is to keep the flood gates closed. In the simulations carried out, about 2.5-3 days were required. One way to reduce the computation time is to use less frequent control updates, e.g., recompute the control input, say, every $24 \mathrm{~h}$ instead of every $1 \mathrm{~h}$. However, we have chosen to use a $1 \mathrm{~h}$ frequency of control updating with the MPC. The following conditions for the simulations are used in the uncertainty analysis.

- $x_{\mathrm{LRV}}=55.75 \mathrm{~m}$ until April 30 , and thereafter $x_{\mathrm{LRV}}=$ $58.85 \mathrm{~m}$.

- $x_{\mathrm{HRV}}=60.35 \mathrm{~m}$ until April 30 , and thereafter $x_{\mathrm{HRV}}=$ $59.85 \mathrm{~m}$.

- $h_{\mathrm{g}}^{(0)}=0 \mathrm{~cm}$; initial gate levels are set to $0 \mathrm{~cm}$.

- $\dot{V}_{\mathrm{i}}$ : inflow forecasts as in Figure 6 and $\dot{W}_{\mathrm{e}}=36 \mathrm{~m}^{3} / \mathrm{s}$.

- $h_{1}$ and $h_{2}$ are set to 59.0 and 58.8 , respectively.

- $N=294$ (length of the operational inflow forecast in hours).

Simulation results are shown in Figures 7, 8, and 9; gate opening $h_{\mathrm{g}, 2}$ is similar to $h_{\mathrm{g}, 1}$. The particles of possible outcomes are represented by gray, dotted lines. The statistics of the outcomes is represented by the mean value (blue line) and standard deviation (yellow area). In this manner, it is possible to link each particle's gate opening with corresponding water level and outflow prediction.

From the figures, it is possible to have an indication of the worst possible situation and take safety measures if needed. In Figure 7, green dashed lines show the upper and lower constraints of levels given by concession requirements. Figure 9 shows both the stochastic behavior of the water flowing through the flood gates $\dot{V}_{\mathrm{g}}$ and the given flow through the turbines, $\dot{V}_{\mathrm{t}}$. As Figure 6 indicates, the inflow forecasts are updated every $24 \mathrm{~h}$.
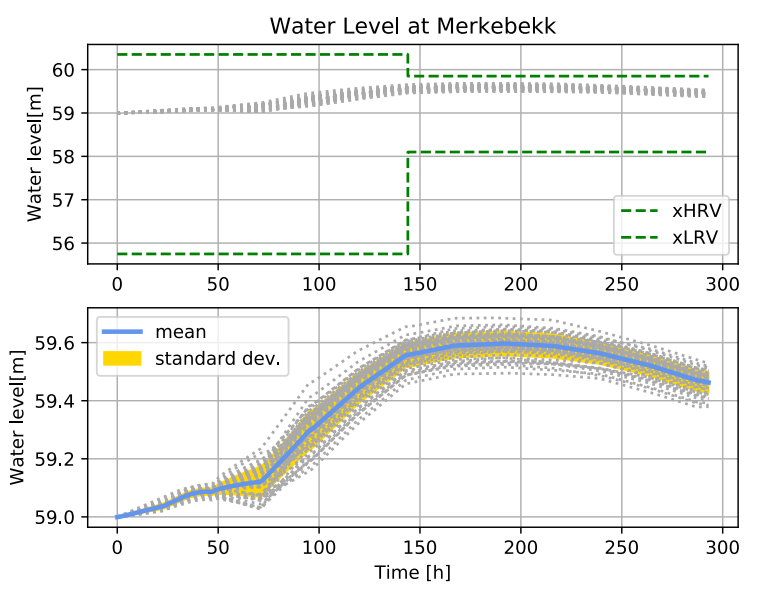

Figure 7. Water Level at Merkebekk for 50 particles with deterministic MPC: lower plot gives a detailed view of upper plot.

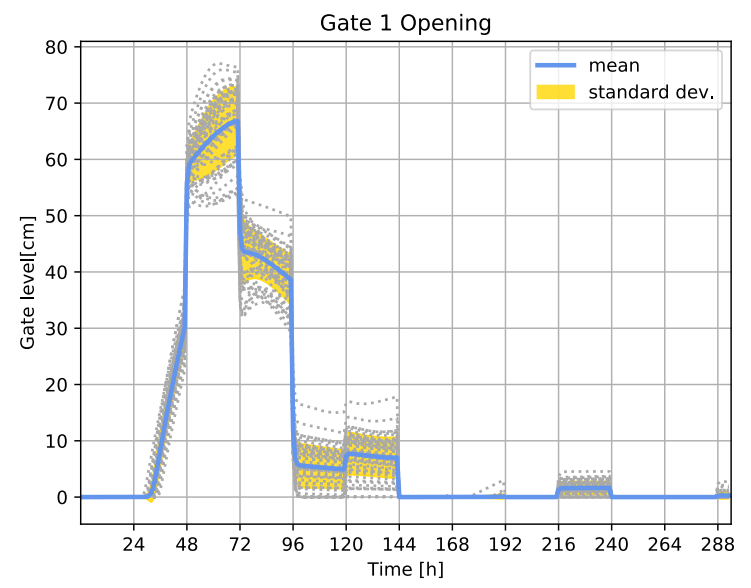

Figure 8. Gate opening $h_{\mathrm{g}}$ for 50 particles with deterministic MPC.

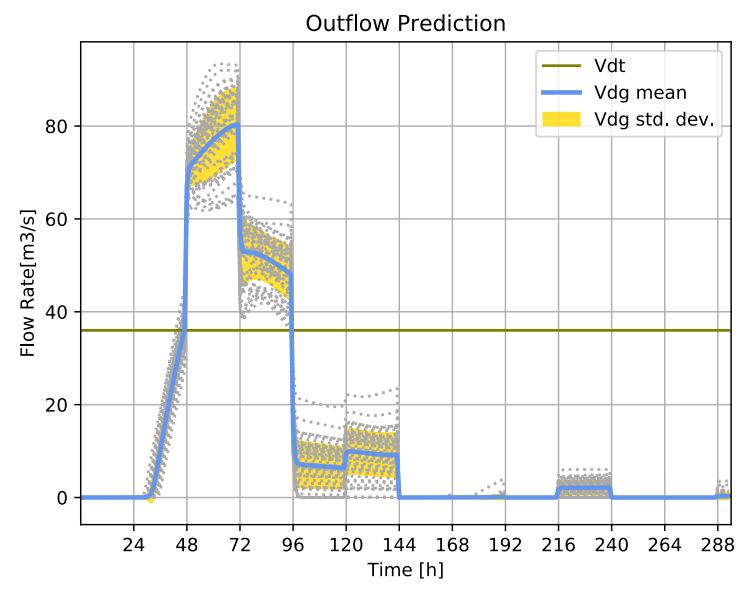

Figure 9. Flow out $\dot{V}_{\mathrm{g}}$ and $\dot{V}_{\mathrm{t}}$ for 50 particles with deterministic MPC. 


\section{Stochastic MPC}

\subsection{Stochastic MPC approach}

Stochastic MPC algorithms can be posed in different ways, e.g., as scenario tree based algorithms (Raso et al., 2014), (Krishnamoorthy et al., 2018), or as multi objective based algorithms (Peitz and Dellnitz, 2018). Here, the focus is on a multi objective based algorithm.

Multi objective optimization (MOO) is based on trying to satisfy a set of objectives with their individual constraints, at the same time. MOO is often a way to find solutions as a best compromise among competing objectives, in a Pareto front manner, e.g., (Boyd and Vandenberghe, 2018). A simple way to handle MOO is to create a combined objective as a scalar weighing of all objectives, while simultaneously satisfying all constraints, (Marler and Arora, 2010), (Sharma, 2017). In the case of our MPC problem, we create an objective $\mathscr{J}_{t}^{(p)}$ for each of the particles $p$ (each column) in the inflow matrix of 12 . Our formulation has only constraints on the gate opening, $h_{\mathrm{g}}^{(p)}$. There is no reason to say that one objective is more important than the others, so we simply sum the objectives for each particle to set up the total objective,

$$
\mathscr{J}_{t}=\sum_{p} \mathscr{J}_{t}^{(p)} .
$$

The constraints are given by the models with each individual inflow particle $\dot{V}_{\mathrm{i}}^{(p)}$, and we require that the control $\operatorname{input} h_{\mathrm{g}}^{(p)}$ is the same for each particle, i.e., $h_{\mathrm{g}}^{(1)}=\ldots h_{\mathrm{g}}^{(50)}$.

\subsection{Simulation results}

The MOO algorithm can use up to 20 min for each optimization under a flood situation. If implemented in in a real time system, this would imply a 20 min time delay in the control loop would reduce the performance of the controller. In our simulation study, we may neglect this time delay. Simulating the system with hourly sampling time for a $294 \mathrm{~h}$ horizon, this implies about $100 \mathrm{~h}$ (4d) of simulation time

The conditions for the experiment are as in Section 3.2. With this stochastic MPC algorithm, the operational inflow is still used as the prediction of inflow in the cost functions $\mathscr{J}_{t}^{(p)}$, and the total cost function $\mathscr{J}_{t}$ is found by summing over all particles $p$ as in 14. This time, the operational input is also used to represent "reality", i.e., in updating the real water levels according to

$$
x_{t+1}=f\left(x_{t}, u_{\mathrm{c}, t}, u_{\mathrm{d}, t}^{(\mathrm{o})}\right),
$$

where $u_{\mathrm{d}, t}^{(\mathrm{o})}=\dot{V}_{\mathrm{i}, t}^{(\mathrm{o})}$, while input $u_{\mathrm{c}, t}$ is the input computed from optimizing $\mathscr{J}_{t}$ according to 14 with initial value $x_{t}$ and operational input predictions $\dot{V}_{\mathrm{i}, t+i}^{(\mathrm{o})}$.

The water level result is shown in Figure 10; the blue curve represents the water level at Merkebekk for which the concession requirements are imposed, while the red curve is the water level at Dalsfos. Likewise, the gate opening result can be seen in Figure 11, with $h_{\mathrm{g}, 1}$ (blue line) and $h_{\mathrm{g}, 2}$ (red line). The predicted outflow result is described in Figure 12, with $\dot{V}_{\mathrm{t}}$ (red line), $\dot{V}_{\mathrm{g}}$ (blue line), and $\dot{V}_{\mathrm{o}}=\dot{V}_{\mathrm{t}}+\dot{V}_{\mathrm{g}}$ (yellow line).

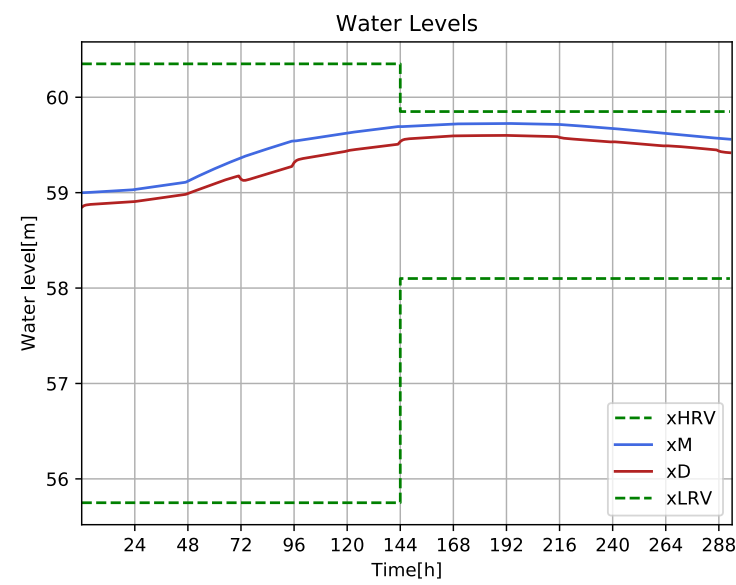

Figure 10. Predicted Water Levels.

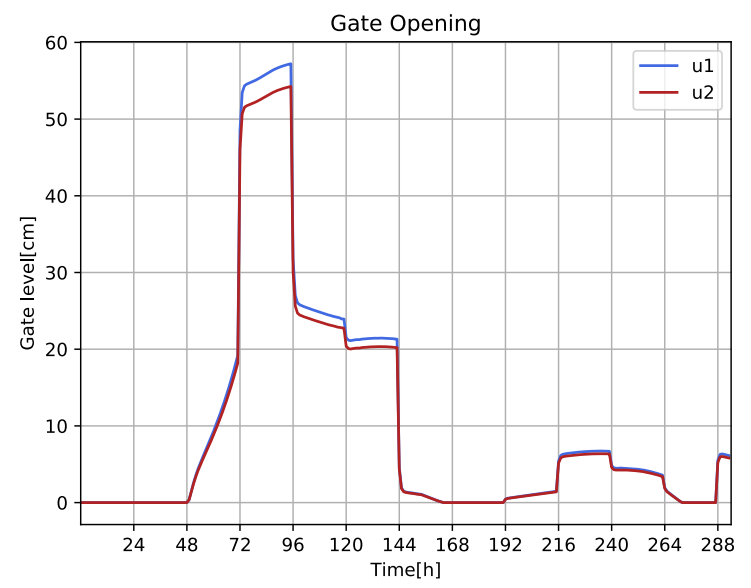

Figure 11. Optimal Gate Openings.

\section{Deterministic vs. Stochastic MPC}

In both the deterministic case and the MOO case, Figure 6 illustrates the inflow disturbances. In the deterministic approach, the operational inflow is the "deterministic" disturbance used in the MPC algorithm while each of the particles are used to compute corresponding water levels. By contrast, in the MOO stochastic MPC approach, the MPC algorithm uses all particles, while the operational inflow is used to compute water levels.

Figures 13-16 illustrate the difference in behavior of the deterministic controller vs. that of the stochastic controller; in the legends, variable $\mathrm{x}$ mean indicates the mean value of variable $x$ for the particles in the deterministic MPC solution, while $\mathrm{x} \_$MOO simply is variable $\mathrm{x}$ for 


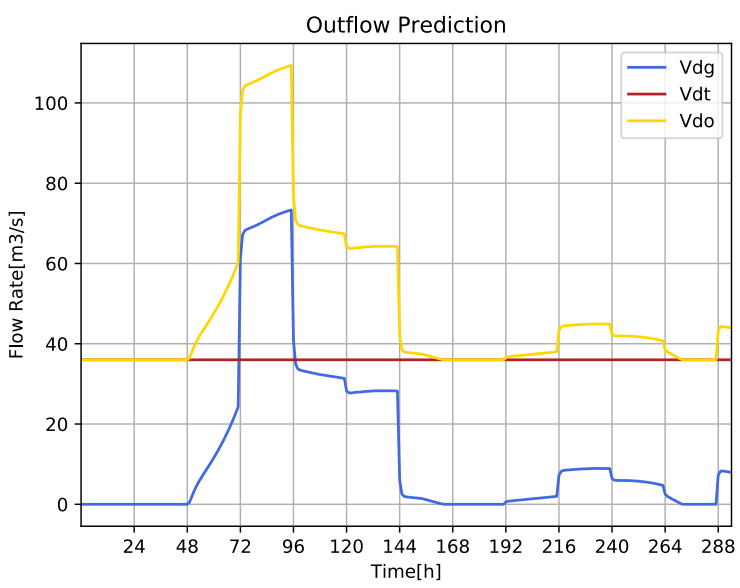

Figure 12. Predicted outflow.

the stochastic multi objective optimization based (MOO) MPC algorithm.
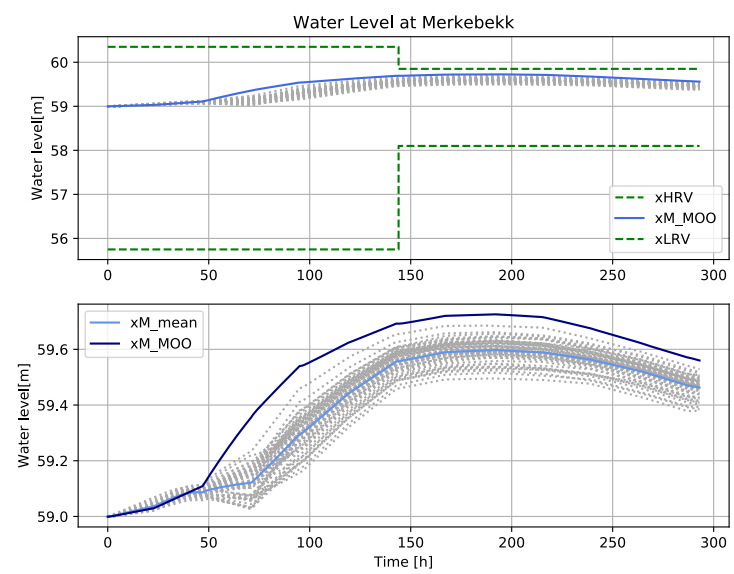

Figure 13. Merkebekk water level $x_{\mathrm{M}}$ comparison: lower plot gives a detailed view of upper plot.

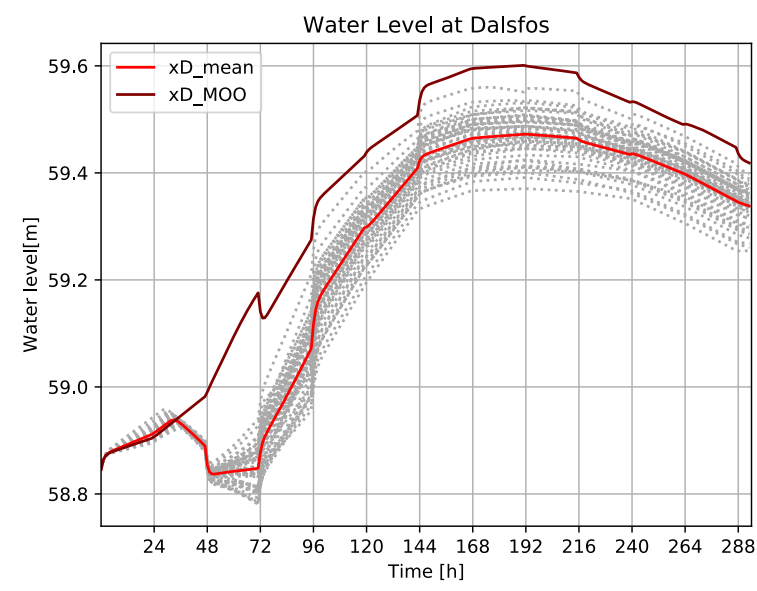

Figure 14. Dalsfos water level $x_{\mathrm{D}}$ comparison.

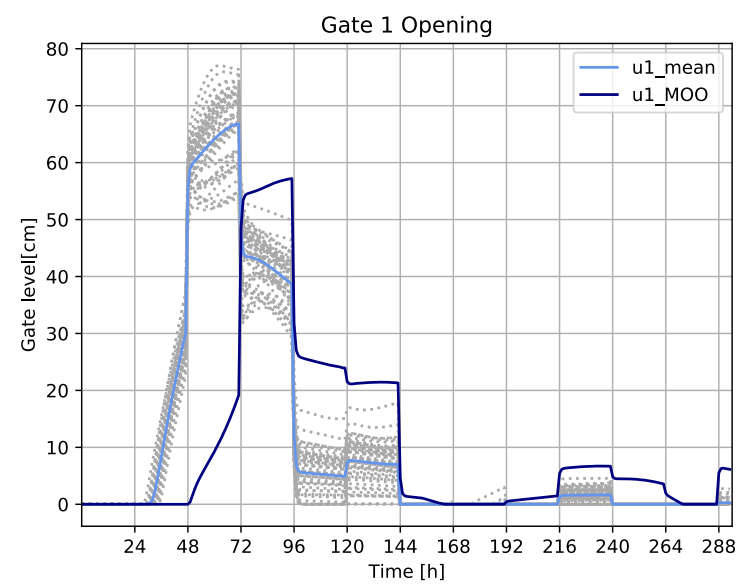

Figure 15. Optimal gate opening $h_{\mathrm{g}, 1}$ comparison.

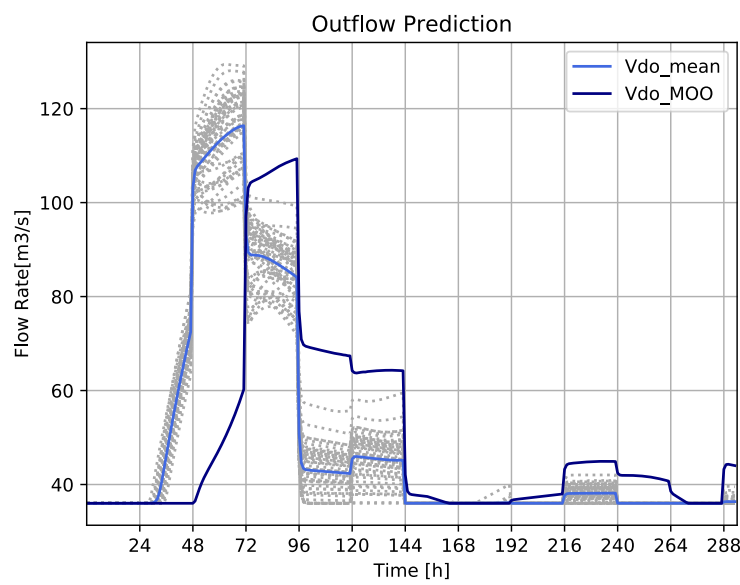

Figure 16. Predicted outflow $\dot{V}_{\text {o }}$ comparison.

Figure 13 displays the predicted water levels at Merkebekk. The dark blue curve (xM) refers to the water level for the MOO case. The gray dotted curves together with the light blue curve ( $\mathrm{xM}$ _mean) corresponds to the water level result from the deterministic approach. In the simulated case, none of the constraints are broken.

Figure 14 displays the predicted water levels at Dalsfos. The dark red curve $(x D)$ refers to the water level for the MOO case. The gray dotted curves together with the light red curve (xD_mean) corresponds to the water level result from the deterministic approach.

Figure 15 illustrates the resulting gate opening $h_{\mathrm{g}, 1}$. The dark blue curve $(u 1)$ refers to the gate opening for the MOO case. The gray dotted curves together with the light blue curve (u1_mean) corresponds to the deterministic approach.

Figure 16 shows the predicted outflow. The dark blue curve $(\mathrm{Vdo})$ refers to the water flow rate for the $\mathrm{MOO}$ case. The gray dotted curves together with the light blue curve (Vdo_mean) corresponds to the flow rate result for the deterministic approach. As expected, the behavior is consistent with the gate openings. 


\section{Conclusions}

The stochastic MOO MPC gives markedly better results than the current, deterministic MPC, indicating a clear advantage in introducing a more advanced stochastic MPC algorithm. It should be noted that the study reported here is limited since only a single instance of real inflow predictions and measurements under flood conditions has been available. Furthermore, it has been assumed that the control input is recomputed every $1 \mathrm{~h}$ and injected every $1 \mathrm{~h}$ without delay. In reality, with manual injection of the computed control inputs, these may be injected irregularly and with time delay. This manual injection of the control input will lead to a more pronounced effect of the uncertainty. Thus, in real life, a stochastic MOO MPC algorithm may be even more advantageous than what appears in the comparison of Section 5.

It is therefore recommended to further the study initiated here, with other flood periods. In particular, the operational forecast may lie in the mainstream of the ensemble as in Figures 5 and 6; other times, the operational forecast lies at the outskirts of the ensemble: the location of the operational forecast within the ensemble may influence the relative advantage of using stochastic MOO MPC. The reported computation time for finding the control signal in the MOO algorithm can be significantly reduced. Examples of strategies to reduce the computation time are utilizing parallelization over threads, more efficient Python code, compiled code, etc.

\section{References}

Stephen Boyd and Lieven Vandenberghe. Introduction to Applied Linear Algebra. Vectors, Matrices, and Least Squares. Cambridge University Press, 2018. ISBN 978-1316518960.

Maarten Breckpot, Oscar Mauricio Agudelo, and Bart L.R. De Moor. Flood Control with Model Predictive Control for River Systems with Water Reservoirs. Irrigation and Drainage Engineering, 139:532-531, 2013a. doi:10.1061/(ASCE)IR.1943-4774.0000577.

Maarten Breckpot, Oscar Mauricio Agudelo, Pieter Meert, Patrick Willems, and Bart De Moor. Flood control of the Demer by using Model Predictive Control. Control Engineering Practice, 21(12):1776-1787, December 2013b. doi:10.1016/j.conengprac.2013.08.008.

Michael B. Butts, Anne Katrine V. Falk, Yunqing Xuan, and Ian D. Cluckie. Integrating meteorological and uncertainty information in flood forecasting: the FLOODRELIEF project. IAHS, 313:385-397, July 2007.

Dines Krishnamoorthy, Bjarne Foss, and Sigurd Skogestad. A distributed algorithm for scenario-based model predictive control using primal decomposition. IFAC PapersOnLine, 51 (18):351-356, 2018

Bernt Lie. Final report: KONTRAKT NR INAN-140122 Optimal Control of Dalsfos Flood Gates- control algorithm, 2014.
R. Timothy Marler and Jasbir S. Arora. The weighted sum method for multi-objective optimization: new insights. Structural and Multidisciplinary Optimization, 41(6):853862, Jun 2010. ISSN 1615-1488. doi:10.1007/s00158009-0460-7. URL https://doi.org/10.1007/ s00158-009-0460-7.

Hasan Arshad Nasir, Tony Zhao, Algo Carè, Quan J. Wang, and Erik Weyer. Efficient river management using stochastic mpc and ensemble forecast of uncertain in-flows. IFAC PapersOnLine, 51(5):37-42, 2018. doi:10.1016/j.ifacol.2018.06.196.

NVE. The Norwegian Water Resources and Energy Directorate, 2018. URL https://temakart.nve.no/link/ ? link=vannkraft \&layer $=0,8 \&$ field $=k d b N r \&$ value $=7332 \&$ buffer $=3000$.

Sebastian Peitz and Michael Dellnitz. A survey of recent trends in multiobjective optimal control - surrogate models, feedback control and objective reduction. Mathematical and Computational Applications, 23(30):1-33, 2018. ISSN 22978747. doi:10.3390/mca23020030. URL http: //www . mdpi.com/2297-8747/23/2/30.

Luciano Raso, Dirk Schwanenberg, Nick van de Giesen, and Peter Jules van Overloop. Short-term optimal operation of water systems using ensemble forecasts. Advances in Water Resources, 71:200-208, September 2014. doi:10.1016/j.advwatres.2014.06.009.

Dirk Schwanenberg, Fernando Mainardi Fan, Steffi Naumann, Julio Issao Kuwajima, Rodolfo Alvarado Montero, and Alberto Assis dos Reis. Short-Term Reservoir Optimization for Flood Mitigation under Meteorological and Hydrological Forecast Uncertainty. Water Resour Manage, 29(5):16351651, March 2015. ISSN 0920-4741. doi:10.1007/s11269014-0899-1. URL https://link.springer.com/ article/10.1007/s11269-014-0899-1.

Roshan Sharma. Lectures notes for course IIA 4117: Model Predictive Control, 2017. 